\title{
Understanding Cognitive And Meta-Cognitive Processes In Construction Management: The System Dynamics Perspective
}

\author{
Amlan Mukherjee', Eddy M. Rojas ${ }^{2}$ and William D. Winn ${ }^{3}$ \\ ${ }^{1}$ Doctoral Candidate, Department of Civil and Environmental Engineering, University \\ of Washington, Seattle \\ ${ }^{2}$ Associate Professor, Department of Construction Management, University of \\ Washington, Seattle \\ ${ }^{3}$ Professor, College of Education, University of Washington, Seattle
}

\begin{abstract}
The fragmented and de-contextualized nature of the construction management (CM) curriculum does not adequately prepare students for the industry. In order to create a more appropriate $\mathrm{CM}$ curriculum, we feel it is imperative to examine the nature of learning in the CM domain. How do novice construction managers learn? How do they build expertise through experience? Most importantly, what tools do we have that will allow us to educate novice construction managers and provide instructors with guidance in answering these questions? We have argued that the CM domain can be modeled using system dynamics. Based on our observation of the students interacting with the Virtual Coach, a situational simulation environment, we have concluded that students in $\mathrm{CM}$ learn by developing a better understanding of the inter-relationships of the sub-components of the CM system (cost, schedule, and resource availability) and its evolution over time. We also contend that tools like the Virtual Coach can be very useful in helping us better educate construction managers.
\end{abstract}

\section{Keywords}

System dynamics, systems thinking, cognition, education, situational simulation

\section{Introduction}

As experienced construction managers retire, a void is left in the construction industry that cannot be easily filled in by recent graduates because of their lack of experience and comprehensive understanding of the domain. In addition, there is a concern in academic circles that the fragmented and de-contextualized nature of the civil engineering/construction management (CE/CM) curricula (McCabe et. al. 2000, Sawhney et al. 2001) does not adequately prepare students for the industry. In this paper we argue that in order to effectively educate CM students to face real world scenarios and be more prepared for the industry, it is imperative to understand learning as a cognitive activity and how it happens in the $\mathrm{CM}$ domain. We propose 
the adoption of a systems perspective in better understanding cognition and metacognition in CM.

As explained by Richmond (1994), systems thinking can be explained as a paradigm and a learning method. The paradigm provides us with a "vantage point" of view of the domain and a set of thinking skills, which focus on understanding the underlying structure of a system in terms of reciprocal inter-relationships of the components and how they unfold in time. The learning method supports the paradigm. We have used the Virtual Coach, a situational simulation of the CM domain, as our method to explore the usefulness of employing the systems thinking paradigm in CM.

In the following sections we explore the field of system dynamics and the systems approach to human cognition to support the claim that it is important to introduce the systems perspective in our understanding of learning in the CM domain. We have also tested 19 students using the Virtual Coach to verify our claim and to show the usefulness of tools like this one in educating CM students.

\section{The systems perspective: System Dynamics /systems thinking}

It is important to briefly examine the "systems perspective" and its origins in the domains of System Dynamics / Systems Thinking (SD/ST). A system is defined as a group of interacting, interrelated, or interdependent elements acting as a complex whole. A complex system is one in which the elements interact to create multi-loop non-linear feedback. Most social systems can be classified as complex systems. Jay Forrester, considered to be the father of the field of system dynamics, defines it as a professional field that combines the theory, methods, and philosophy needed to analyze the behavior of complex systems using a common foundation that can be applied whenever we want to understand and influence the change of behavior over time (Forrester 1991, 1999). System dynamics involves interpreting real life systems into computer simulation models that allow us to understand how the structure and decision-making policies in a system create its behavior.

Richmond (1991, 1994) has defined "systems thinking" as the art and science of making reliable inferences about behavior by developing an increasingly deep understanding of underlying structure. He explains that the systems thinking is "system dynamics with an aura," that is, it provides a layman's approach to understanding the emergent behavior of complex systems, without being intimidated by the mathematical methodologies employed in analyzing system dynamics.

Richmond (1991) further goes on to explain that SD/ST in practice is a continuum of activities, which range from the conceptual to the technical. On the far left is the purely conceptual systems viewpoint, that is arrived at by "standing back far enough" from a system in both space and time to see the "underlying web of ongoing, reciprocal relationships which are cycling" to produce patterns of behavior. As we proceed rightwards along the continuum, the emphasis shifts toward implementing the viewpoint and becomes more analytical. This involves the use of influence diagrams and formal models to conceptualize and eventually mathematically express the inter-relationships and feedback loops that are present in the system. Finally, the formal mathematical models can be used to power simulations that can allow us to 
simulate and verify the models, explore "what-if" scenarios and forecast emergent behavior of the system.

The main advantage of using a SD/ST approach in order to understand a domain is that it allows researchers to "stand back" and be able to adjudge the impacts of events and decisions that are often not limited locally in time. It also helps in developing solutions to problems by getting a better understanding of the feedbacks and counter-actions that occur because of the immediate problem at hand. Forrester (1971) analyzes the counter-intuitive behavior of social systems, and explains that orderly processes in creating human judgment and intuition lead people to counterintuitive decisions arising out of a conflict between the goals of a component of the system and its greater good. Sterman (1992) explains why the domain of CM is counterintuitive. For example, a delayed project tends to get even more delayed when more resources are added to it. This kind of behavior is typical with respect to complex and highly interacting systems.

The construction management domain can be studied as a complex system, which has multiple interacting components (schedule, cost, resource distribution and availability, etc.) with multiple feedback loops. Using the SD/ST approach to model CM projects is not an entirely new idea. Sterman (1992) correctly asserts that attributes of construction projects are complex, consisting of multiple inter-dependent parts, involving multiple feedback processes and non-linear relationships. He explains that system dynamics can be used to capture the interdependencies in the CM domain so that causal impact of changes can be traced throughout the system.

\section{Cognition and the Systems Approach: How People Learn}

The prevalent approach to understanding how people learn has been the computational approach to human cognition. Such an approach says that knowledge about the world is represented in memory by static structures of discrete symbols, and all cognitive operations (learning and decision making) are essentially discrete, sequential and instantaneous transformations from one structure to the next. However, criticisms that cognitive activity is contextually situated (Brown, Collins and Duguid, 1989) and is not simply a mapping of external events to an internal symbolic system (Maturana and Varela 1987) has led researchers to study the context and culture in which cognition occurs (Winn 2002). Recently, the constructivist school of thought has explained learning in terms of students evolving to a greater contextualized understanding of their experiential world. It holds that learning is a process in which individuals construct their own meanings of the world they observe, and that the psychological processes involved are "essentially the same as the epistemological processes by which new knowledge is constructed by professionals in a discipline" (Novak 1993). However, critics of constructivism argue that it borders "towards relativism, or towards treating the justification of our knowledge as being entirely a matter of socio-political processes or consensus, or towards the jettisoning of any substantial rational justification or warrant" (Phillips 1995).

Winn (2002) provides an alternative framework for describing learning in artificial environments, based on the three concepts of embodiment, embeddedness and adaptation. One of the implications of the framework is that it couples the learner and the environment into "one evolving system rather than two interacting ones." 
Learning, thus, can be thought of as a "self-organization by the system and new knowledge as an emerging property of that self-organizing activity." It allows us to conclude that a successful learning environment in construction would have to conceive the learner and the environment as a coupled system.

Meanwhile, Port and Van Gelder (1995) have also argued that the problem with the computational approach to cognition is that it fails to recognize that "cognitive processes and their contexts unfold continuously and simultaneously in real time." The authors further go on to state that decision-making problems cannot be expressed completely within the computational model of human cognition because it does not take into account changes in behavior of parameters and components over time. There is no explicit representation of time beyond it being a sequence of discrete events. It also does not take into account the effect of the time spent in the deliberation process on the decision itself. They finally conclude that an alternative approach to understanding cognitive processes is by treating cognitive systems as dynamical systems.

This echoes Winn's (2002) notion of the learner and the learning environment being a coupled system and strengthens the argument that human cognition is not only contextualized and adaptive, but also dynamic. Knowledge is an emergent property of the coupled dynamical system consisting of the learner and the learning context. This is particularly significant with respect to the CM domain, because we cannot deny the critical role of human decision-making in it.

The success of a construction project (a system which evolves from start to completion) in terms of time and budget is dependent on the skill of the construction manager (the learner in our environment). As students learn within the environment, their performances improve and directly affect the evolution of the environment itself. Hence, a learning environment for the $\mathrm{CM}$ domain that aims at bridging disconnect between fragmented presentation of theory and practice in CM courses needs to be interactive and adaptive and it should present the CM domain to the students as a dynamical system. This would facilitate and aid the process of learning by helping students cognitively better understand the systemic nature of the CM domain.

\section{The Virtual Coach}

The Virtual Coach is a situational simulation environment for the construction management domain. A Situational simulation is a temporally dynamic, interactive and adaptive simulation. It is supported by a well-defined mathematical model (Rojas and Mukherjee 2003) that captures underlying systemic inter-relationships between cost, schedule and resources in the $\mathrm{CM}$ domain. Implementations of situational simulations, such as the Virtual Coach, use cost and schedule information for the simulated project; and a knowledge base containing rules pertinent to the domain and the project. The situational simulation environment is controlled and generated by collaborating autonomous agents (Mukherjee and Rojas 2003a), which create events as consequences of user interaction and also based on the context of the simulation using a statistical event generator.

A situational simulation runs in quasi real time (i.e., the time taken by the project being simulated in real life is some integral multiple of the simulated time). Participants are required to interact with the simulation environment and manage the 
simulated project to successful completion. They control the evolution of the simulation by managing resources and making critical decisions to apprehend and manage events that may delay the project. For instance, in a situational simulation of an earth moving operation participants might be in a situation where they are required to finish the activity at hand within budget and time constraints under the influence of bad weather. The autonomous agents running the simulation maintain consistency of the temporal and resource constraints that capture the systemic relationships between costs, schedule and resource allocation. Using the underlying mathematical model and logical reasoning, the agents can reflect the impacts of constraint violations resulting from participants' decisions. This allows participants to comprehend the long term impacts of their decisions.

The pilot implementation of the Virtual Coach simulates a twelve activity hypothetical project with realistic constraint violations and event information. Participants have the ability to allocate, reallocate, or procure resources from the market place. They are presented with the challenge of quick decision-making amidst rapidly unfolding events. By exploring 'what-if' scenarios, participants can also test the sensitivities of the system to their decisions in a dynamic environment. At the end of each simulated 'week' the program creates a progress report with information regarding the current state of the schedule as compared to the "As-Planned" schedule. Such information helps participants in monitoring their progress and perceiving the system dynamics of the CM domain. The final goal of participants is to steer the project through generated scenarios and complete it within budget and time constraints.

\section{Experimentation and Results}

A pilot of the Virtual Coach situational simulation environment was tested with a group of 19 senior level construction management students, as part of a Project Management class at the University of Washington. The students took pre and posttests before and after they experienced the simulation. They were also required to "think aloud" their decisions and their perceptions of what was happening during the exercise. All comments made during the simulation were recorded. The pre-test and post-test required students to rank (on a scale of 1-10), in their opinion, the importance of a list of factors in developing a plan for a 12 week period of a construction scenario. They were also provided with a list of constraints governing the scenario and the necessary project information. The constraints included schedule considerations, budget limitations and the possibilities of events such as bad weather, material delivery delays and labor shortage.

Four of the priority ratings assigned by the students, before and after using the simulation, were summed and compared using a paired-sample t-test. The ratings selected for analysis were those that related to the schedule and resource constraints and the need to anticipate delay on a project (giving priority to critical activities in case of delay, attention to space restrictions on site, anticipating future material delivery delays, accelerating activities to create buffer for anticipated delay, etc.) The difference between the ratings was significantly different: 
Table 1: Student Performance in Pre- and Post-Test

\begin{tabular}{|c|c|c|}
\hline & Mean & Std. deviation \\
\hline Pre-Test & 21.26 & 4.92 \\
\hline Post-Test & 25.31 & 4.70 \\
\hline \multicolumn{3}{|c|}{ T-statistic: $\mathrm{t}(18)=3.32, \mathrm{p}<.01$} \\
\hline
\end{tabular}

It is interesting to note that the questions on which the students showed significant improvement dealt with resource or temporal constraint satisfaction suggesting that students learn by understanding the underlying constraints present in a project and apprehending their violations.

A qualitative analysis of the participant's 'thoughts' (as recorded during the simulation) and the feedback provided in a post simulation survey provided us with valuable insight into what the students learnt from the experience. Below is a list of selected reactions from the students after the simulation was over. They have been reported verbatim and are representative of the general feedback that we got from the students. The highlighted sections of the responses emphasize the fact that what impressed the students most was the ability to get the "bigger picture" and the interrelationships between labor, budget, schedule and the impact of their decisions on the environment.

Participant 1: I liked the fact that I was able to see what my actions were doing to the budget and schedule. In the industry you are always trying to pick up a few critical days in the beginning to counteract unforeseen setbacks in the future.

Participant 2: Virtual coach was a good simulation and put together the critical elements of managing a project, labor, materials, schedule, and cost. I feel that it provides a good way of actually controlling a schedule and seeing the effect one change can have on all the varaibles.

Participant 3: I like how the project did not go according to plan. I think it was a good way to communicate as to how unforseen events happen thus you need to change the way in which you approach your already existing constraints.

Participant 4: The virtual coach does a good job with giving students a better idea of the big picture. . . . I felt like I needed to understand how the relationships between the material and labor allocation were determined and being used before I really put a lot of trust into the Virtual Coach.

Participant 5: I thought the Virtual Coach is really interesting in the fact that it accounted for the many outside parameters that may affect the construction process of a project.

Participant 6: The real time cost allocation and schedule allows one to see where they are at and where they are going is great. 
Participant 7: I think that virtual coach is a great activity for us to use. it gets you to think about resource, management, estimating and scheduling together. I think it could be improved by letting us see the differences in unit costs, and how much our discisions affect the project. for example, it would have lice to see how much more expensive it is to hire labor at a premium. other then that it is a great program, any schoolwork I have done so far has not taught resource managing as well as this has.

Participant 8: Virtual coach did an excellent job of forcing me to see the big picture or suffer the consequences of lost productivity, lost \$, etc. I feel that the scenarios and interaction with the program as far as the percentage of likelyhood of certain events was fairly true. For example, if I worked the workers too hard they would be more likely to perform poor work or strike.

Participant 9: I thought Virtual Coach was an educating experience. I thought that it showed and allowed the students to have a good understanding of the decisions they made and how the decidions influenced the schedule and the budget.

Participant 8 also emphasizes the bigger picture and recognizes some simple dependencies that create the underlying structure in the CM domain. Participant 7 suggests that the program should be further developed to reflect in greater detail the sensitivity of their decisions on the project. Clearly this is an example of the students having realized that "resource, management, estimating and scheduling" are related, function together in a system, and therefore it is necessary to evaluate the sensitivity of the system to the each decision.

Participant 4's response also highlights the understanding gained of the "bigger picture" and the "understand the relationships" between material and labor allocation. It is also very encouraging to note that the student feels the need to know the workings of the simulation model.

The recorded reactions of participants during the simulation helped us detect the various 'Ah-ha' moments that reinforce the belief that the simulation was helping students achieving a systemic view of the CM domain. It was very interesting to note how the students were reacting to delays in the schedule. The most common reaction was to accelerate the activity at hand, without paying attention to where it was on the critical path. There was also a general tendency to increase the productivity of labor on a delayed activity without anticipating a feedback in terms of rework or labor unavailability in the future. It was interesting to note a student comment when he realized that a project impacted by an event toward the end of the schedule is a lot more difficult to recover from than a project delayed early on.

The 'Ah-ha' moments happened when the unexpected feedback came back to make the user rethink a decision taken earlier on in the simulation. The understanding that there exist 'lag times' between action and feedback motivated the students to "stand back" and get a bigger picture of how sensitive the system was to their decisions. It also helped them perceive that problems are often not just localized disturbances, but results of structural causal relationships, which are reciprocal in nature (Participant 8). 


\section{Discussion and Conclusion}

Based on qualitative feedback (post simulation survey) from the students, where 16 out of 19 thought that the Virtual Coach was a useful educational tool; the statistical significance of the post- and pre-test results; and the high differential values of the confidence interval as illustrated in Table 1; we can conclude that an intervention using situational simulations can be useful in construction education.

From our study we also conclude that students tend to learn better by understanding the underlying constraints present in a project and apprehending their violations while making decisions. Our analysis shows that the way students feel better equipped to apprehend such constraint violations is by getting a systemic view of the CM domain and a better understanding of the inter-relationships and causal loops. Based on the enthusiastic feedback we received from the students and our analysis of their reactions, we strongly encourage further development of adaptive and dynamical learning environments, such as the Virtual Coach, as useful tools in $\mathrm{CM}$ education. Furthermore, given the body of literature in the SD/ST and the cognitive science community, which support the usefulness of such learning methods, we strongly encourage the adoption of such environments into the CM curriculum. This does not mean that we abandon traditional classroom teaching methods. Instead, we should harness the power of such environments and the SD/ST approach to enhance understanding. It is not an "either or" and indeed it would be unfortunate if we lost the joy of listening to the leaves rustle in our quest for better understanding the forest.

Finally, how does this understanding stand to influence the CM domain beyond education? The answer lies in our understanding of the meta-cognition involved in CM. What do we learn about the domain from our knowledge of how we learn in it? This is not an easy question to answer conclusively within the scope of this paper. However, we would like to suggest that the use of situational simulations may help us better research this question and develop a theoretical understanding of construction without assuming it to be akin to other industries like manufacturing (Koskela and Vrijhoef 2001).

\section{References}

Brown, J.S., Collins, A., and Duguid, P. (1989). "'Situated cognition and the culture of learning," Educational Researcher 1989 18(1), 32-42.

Forrester, J.W. (1971). "Counterintuitive Behavior of Social Systems" Technology Review, Alumni Association of Massachusetts Institute of Technology.

Forrester, J.W. (1991) "System Dynamics and the lessons of 35 Years" A Chapter for The Systemic Basis for Policy Making in the 1990s, ed. DeGreen, K.B.

Forrester, J.W. (1994) "Learning through System Dynamics as Preparation to the $21^{\text {st }}$ Century." Keynote address for System Thinking and Dynamic Modeling Conference for k-12 Education, Concord Academy. Concord MA, USA.

Koskela, L. and Vrijhoef, R. (2001). "The Prevalent Theory of Construction is a Hindrance for Innovation," Building Research and Innovation, Vol. 29 Nr:3, pp 197-207. 
Mukherjee, A. and Rojas, E. (2003a) "Reasoning about Actions and Events in Situational Simulations" In Proceedings of Winter Simulation Conference (SIGSIM), December 7th-10th, 2003a, New Orleans, LA.

Maturana, H. and Varela, F. (1987). "The tree of Knowledge” Boston: New Science Library

McCabe, B., Ching, K.S., and Savio, R. (2000). "STRATEGY: a construction simulation environment," Proceedings of Construction Congress VI, ed. Kenneth D. Walsh, pp 115-120.

Novak, J. (1993) "Human Constructivism: A unification of psychological and epistemological phenomena in meaning making." International Journal of Personal Construct Psychology, 6, pp167-193

Phillips, D.C. (1995) "The Good, the Bad and the Ugly: The Many Faces of Constructivism" Educational Researcher, Vol. 24, No. 7, pp 5-12

Port, R.F. and Van Gelder, T.(1995).’Its about time” In R.F. Port and T. Van Gelder, "Mind as Motion: Explorations in the dynamics of Cognition" Cambridge, MA: MIT Press.

Richmond Barry (1991) "Systems Thinking: Four key questions" Copyright, 1991, High Performance Systems, Inc.

Richmond Barry (1994) "System Dynamics /Systems Thinking: Lets Just Get on With It" Delivered at the 1994 International System Dynamics Conference in Sterling Scotland.

Rojas, E., and Mukherjee, A. (2003) "Modeling the Construction Management Process to Support Situational Simulations." Journal of Computing in Civil Engineering, ASCE, 17(4), 273-280

Sawhney, A., Mund, A., and Koczenasz, J. (2001). "Internet-based interactive construction management learning system," Journal of Construction Enducation, 6 (3), 124-138.

Sterman, J.D. (1992) "Systems Dynamics Modeling for Project Management" Systems Dynamics Group, Sloan School of Management. Massachusetts Institute of Technology, Cambridge, MA 02139.

Winn, W.D. (2002). "Learning in Artificial Environments: Embodiment, Embeddedness and Dynamic Adaptation" Tech., Inst., Cognition and Learning Vol 1, 2002 